\title{
Measuring Subjective Cognitive Decline in Older Adults: Harmonization between the Cognitive Change Index and the Measurement of Everyday Cognition Instruments
}

\author{
Lindsey F Wells ${ }^{1}$, Shannon L Risacher ${ }^{2,3}$, Andrew J Saykin ${ }^{2,3}$ \\ ${ }^{1}$ Indiana Medical Student Program for Research and Scholarship \\ ${ }^{2}$ Center for Neuroimaging, Department of Radiology and Imaging Sciences, Indiana University \\ School of Medicine, Indianapolis, IN USA \\ ${ }^{3}$ Indiana Alzheimer Disease Center, Indiana University School of Medicine, Indianapolis, IN USA
}

\section{Background and Hypothesis:}

Self and informant reports of everyday cognitive functioning have been shown to be associated with incipient neurodegenerative disease. The 20-item Cognitive Change Index (CCI) [1] and the 39-item Measurement of Everyday Cognition (ECog) [2] were each developed to characterize early changes in cognitive function. The aim of this study is to examine the relationship between the $\mathrm{CCl}$ and ECog self- and informant-evaluations to improve early detection and longitudinal assessment of cognitive decline for observational research and clinical trials.

\section{Experimental Design or Project Methods:}

Self-evaluation data included 803 participants $(49.3 \%$ male, mean age $=69.9 y r s)$ from the Alzheimer's Disease Neuroimaging Initiative (ADNI), the Indiana Alzheimer Disease Center (IADC), and the Indiana Memory and Aging Study (IMAS). Informant-evaluation data included 288 participants $(39.9 \%$ male, mean age $=66.9 y$ rs $)$ from the IMAS and IADC studies. Study participants from 2012 to 2019 were included if they had completed both the CCl and ECog at the time of their baseline visit. Pearson correlations, regression analyses, and frequency distributions were used to assess the relationship between the $\mathrm{CCl}$ and ECog. Sex, years of education, race, ethnicity, $A P O E \varepsilon 4$ carrier status, and baseline diagnosis were also analyzed as potential relevant covariates.

\section{Results:}

$\mathrm{CCl}$ and ECog total scores were highly correlated for the self $(r=0.778, p<0.001)$ and informant $(r=0.892, p<0.001)$. The highest associations were between the mean item scores for each instrument for both self $(r=0.801, p<0.001)$ and informant $(r=0.913, p<0.001)$. Frequency distributions showed distinct patterns between self and informant total scores. Polynomial regressions for self $\left(R^{2}=0.673\right)$ and informant $\left(R^{2}=0.873\right)$ total scores were used to create a translation table between the $\mathrm{CCl}$ and ECog.

\section{Conclusion and Potential Impact:}

Self and informant total scores can be harmonized and translated between the $\mathrm{CCl}$ and ECog to facilitate cross-sectional and longitudinal assessment of perceived cognitive change, an important patient reported outcome.

[1] Rattanabannakit et al. (2016). Journal of Alzheimer's Disease, 51(4), 1145-1155.

[2] Farias et al. (2008). Neuropsychology, 22(4), 531-544. 